Document downloaded from:

http://hdl.handle.net/10251/104217

This paper must be cited as:

Singh, S.; Gupta, DK.; Badoni, RP.; Martínez Molada, E.; Hueso Pagoaga, JL. (2017). Local convergence of a parameter based iteration with Holder continuous derivative in Banach spaces. CALCOLO. 54(2):527-539. doi:10.1007/s10092-016-0197-9

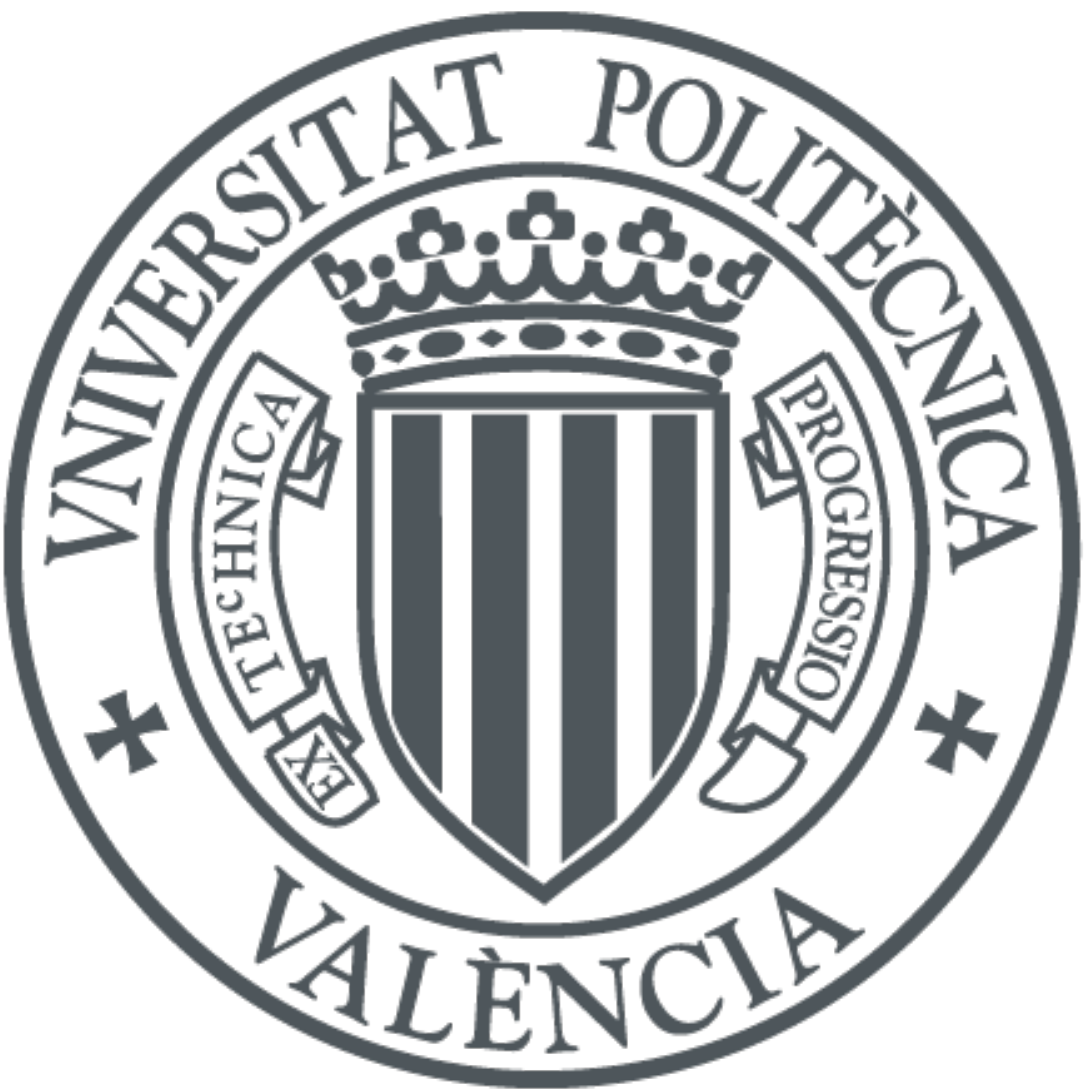

The final publication is available at

http://dx.doi.org/10.1007/s10092-016-0197-9

Copyright Springer-Verlag

Additional Information 


\title{
Local convergence of a parameter based iteration with Hölder continuous derivative in Banach spaces
}

\author{
Sukhjit Singh, D.K. Gupta, Rakesh P. Badoni, E. \\ Martínez, José L. Hueso
}

Received: date / Accepted: date

\begin{abstract}
The local convergence analysis of a parameter based iteration with Hölder continuous first derivative is studied for finding solutions of nonlinear equations in Banach spaces. It generalizes the local convergence analysis under Lipschitz continuous first derivative. The main contribution is to show the applicability to those problems for which Lipschitz condition fails without using higher order derivatives. An existence-uniqueness theorem along with the derivation of error bounds for the solution is established. Different numerical examples including nonlinear Hammerstein equation are solved. The radii of balls of convergence for them are obtained. A substantial improvement of these radii are found in comparison to some other existing methods under similar conditions for all examples considered.
\end{abstract}

Keywords Nonlinear equations $\cdot$ Local convergence $\cdot$ Banach space $\cdot$ Lipschitz condition · Iterative methods $\cdot$ Hölder condition $\cdot$ Hammerstein integral equation

Mathematics Subject Classification (2000) 65G49 • 47H99

\author{
First Author \\ Department of Mathematics, Indian Institute of Technology Kharagpur - 721302, India E-mail: sukhjit- \\ math@gmail.com \\ Second Author \\ Department of Mathematics, Indian Institute of Technology Kharagpur - 721302, India E-mail: \\ dkg@maths.iitkgp.ernet.in \\ Third Author \\ Department of Mathematics, Indian Institute of Technology Kharagpur - 721302, India E-mail: rakesh- \\ badoni@gmail.com \\ Fourth Author
}

Instituto de Matemática Pura y Aplicada, Universitat Politècnica de València E-mail: eumarti@mat.upv.es Fifth Author

Instituto de Matemática Multidisciplinar, Universitat Politècnica de València E-mail: jlhueso@mat.upv.es 


\section{Introduction}

Many problems of applied sciences when modeled mathematically give rise to differential equations, boundary value problems and integral equations whose solutions are obtained by solving nonlinear equations. Static and dynamical systems, optimization problems, Kinetic theory of gases, etc. are other areas giving rise to thousands of such equations. These facts make it one of the most important areas to be studied. Except for very simple types of problems which can be solved analytically, iterations and their local convergence are used for them. Another equally important is to obtain the radii of convergence balls and give hypothesis in order to enlarge them. A number of researchers $[4,5,11,14]$ have extensively studied this problem and a number of methods are developed for solving them. It should be noted that the local convergence analysis $[1,6,16]$ use information around the solution.

Here, we consider approximating a solution $x^{*}$ of

$$
G(x)=0
$$

where, $G$ defined on a subset $D$ of a Banach space $X$ with values in a Banach space $Y$ and having Fréchet derivatives of appropriate orders. The quadratically convergent iteration known as the Newton's method for solving (1.1) is given by

$$
x_{k+1}=x_{k}-G^{\prime}\left(x_{k}\right)^{-1} G\left(x_{k}\right), \quad k \geq 0,
$$

where, $x_{0}$ is the starting point and $G^{\prime}\left(x_{k}\right)^{-1}$ is the inverse of $G^{\prime}\left(x_{k}\right)$. Kantorovich [9] gives sufficient conditions for its convergence. Higher order iterations involve the computation of derivatives of higher orders which are either expensive or unbounded. The third order iterations used for solving (1.1) are the Chebyshev's, the Halley's and Super-Halley's methods obtained by taking $(\alpha=0),\left(\alpha=\frac{1}{2}\right)$ and $(\alpha=1)$ and defined for $k \geq 0$, by

$$
x_{k+1}=x_{k}-\left(1+\frac{1}{2}\left(1-\alpha H_{G}\left(x_{k}\right)\right)^{-1} H_{G}\left(x_{k}\right)\right) G^{\prime}\left(x_{k}\right)^{-1} G\left(x_{k}\right),
$$

where, $H_{G}\left(x_{k}\right)=G^{\prime}\left(x_{k}\right)^{-1} G^{\prime \prime}\left(x_{k}\right) G^{\prime}\left(x_{k}\right)^{-1} G\left(x_{k}\right)$. The drawback of these methods is that they require computation of $G^{\prime \prime}(x)$, which is either unbounded or difficult to compute. The local convergence of an iteration of order five and six [10] for the modification of (1.3) given by [12] is defined for $k \geq 0$, by

$$
\begin{aligned}
y_{k} & =x_{k}-G^{\prime}\left(x_{k}\right)^{-1} G\left(x_{k}\right), \\
z_{k} & =x_{k}\left(I+\left(G\left(x_{k}\right)-2 \alpha G\left(y_{k}\right)\right)^{-1} G\left(y_{k}\right)\right) G^{\prime}\left(x_{k}\right)^{-1} G\left(x_{k}\right), \\
x_{k+1} & =z_{k}+\left(G^{\prime}\left(x_{k}\right)+\hat{G}^{\prime \prime}\left(x_{k}\right)\left(z_{k}-x_{k}\right)\right)^{-1} G\left(z_{k}\right),
\end{aligned}
$$

where, $x_{0}$ is the starting point, $\alpha \in[0,1]$ and $\hat{G}^{\prime \prime}\left(x_{k}\right)=2 G\left(y_{k}\right) G^{\prime}\left(x_{k}\right)^{2} G\left(x_{k}\right)^{-2}$. The deformed Halley's method is given for $k \geq 0$, by

$$
\begin{aligned}
y_{k} & =x_{k}-G^{\prime}\left(x_{k}\right)^{-1} G\left(x_{k}\right), \\
z_{k} & =x_{k}+\alpha G^{\prime}\left(x_{k}\right)^{-1} G\left(x_{k}\right), \\
H_{k} & =\frac{1}{\lambda} G^{\prime}\left(x_{k}\right)^{-1}\left[G^{\prime}\left(x_{k}+\lambda\left(z_{k}-x_{k}\right)\right)-G^{\prime}\left(x_{k}\right)\right], \\
x_{k+1} & =y_{k}+\frac{1}{2} H_{k}\left(I-\frac{1}{2} H_{k}\right)^{-1}\left(y_{k}-x_{k}\right),
\end{aligned}
$$


where $\lambda \in(0,1], \alpha \in \mathbb{R}$ and $x_{0}$ is the starting point. Its local convergence analysis is presented in [13] under Hölder condition on $F^{\prime}$. It avoids computation of $G^{\prime \prime}(x)$. Many other similar methods avoiding $G^{\prime \prime}(x)$ are also described. Thus, higher order iterations have their own importance in real life applications. For example, stiff system of equations which require quick convergence. The local convergence discussed in [3] of a family of iterations of third order with Lipschitz continuous $G^{\prime}$ is given by

$$
\begin{aligned}
y_{k} & =x_{k}-G^{\prime}\left(x_{k}\right)^{-1} G\left(x_{k}\right), \\
x_{k+1} & =x_{k}-\frac{\theta^{2}+\theta-1}{\theta} G^{\prime}\left(x_{k}\right)^{-1} G\left(x_{k}\right)-\frac{1}{\theta^{2}} G^{\prime}\left(x_{k}\right)^{-1} G\left(y_{k}\right), k \geq 0,
\end{aligned}
$$

where, $\theta \in \mathbb{R}-\{0\}$ and $x_{0}$ is the starting point. The local convergence of multi-point-parametric Chebyshev-Halley-type methods of higher orders for solving (1.1) is discussed in [7] under Lipschitz continuous $G^{\prime}$ in Banach spaces. The local convergence of a modified Halley-Like method of high convergence order under Lipschitz continuous $G^{\prime}$ is established in [8].

In this paper, the local convergence analysis of a parameter based iteration with Hölder continuous first derivative is studied for finding solutions of nonlinear equations in Banach spaces. It generalizes the local convergence analysis under Lipschitz continuous first derivative. The main contribution is to show the applicability to those problems for which Lipschitz condition fails without using higher order derivatives. An existence-uniqueness theorem along with the derivation of error bounds for the solution is established. Different numerical examples including nonlinear Hammerstein equation are solved. The radii of balls of convergence for them are obtained. A substantial improvement of these radii are found in comparison to some other existing methods under similar conditions for all examples considered.

The arrangement of this paper is done as follows. Introduction forms Section 1. In Section 2, the local convergence analysis of a family of iterations is established under Hölder condition on $G^{\prime}$ in Banach spaces. The existence and uniqueness theorem is established. A number of different numerical examples including a nonlinear Hammerstein integral equation from the kinetic theory of gases are worked out in Section 3. The local convergence of the same family is studied with the condition that involves $M>0$ in Section 4. Finally, conclusions are included in Section 5 .

\section{Local convergence}

A family of iterations [15] and its local convergence to solve (1.1) is established under Hölder continuous $G^{\prime}$. It is given for $k \geq 0$, by

$$
\begin{aligned}
y_{k} & =x_{k}-a G^{\prime}\left(x_{k}\right)^{-1} G\left(x_{k}\right), \\
z_{k} & =y_{k}-G^{\prime}\left(x_{k}\right)^{-1} G\left(y_{k}\right), \\
x_{k+1} & =z_{k}-\left(\frac{1}{a} G^{\prime}\left(y_{k}\right)^{-1}+\left(1-\frac{1}{a}\right) G^{\prime}\left(x_{k}\right)^{-1}\right) G\left(z_{k}\right),
\end{aligned}
$$

where, $a \in(-\infty, \infty)-\{0\}$ and $x_{0}$ is the starting point. Its order of convergence is five for $a= \pm 1$, otherwise it is fourth order. This is the extension of [2], where its local convergence is established under Lipschitz continuous $G^{\prime}$. Examples can be constructed for which Lipschitz condition fails but Hölder condition holds on $G^{\prime}$.

Example 1 Consider an integral equation given by

$$
G(x)(s)=x(s)-3 \int_{0}^{1} G_{1}(s, t) x(t)^{5 / 4} d t,
$$

with $x(s) \in \mathcal{C}[0,1]$ and $G_{1}(s, t)$ is the Green function. 
Then,

$$
\left\|G^{\prime}(x)-G^{\prime}(y)\right\| \leq \frac{15}{32}\|x-y\|^{1 / 4} .
$$

Clearly, Lipschitz condition fails on $G^{\prime}$ but Hölder condition holds for $p=\frac{1}{4}$. Let $M_{1}>0$, $M_{2}>0, p \in(0,1]$. Let $B L(Y, X)$ represents the set of bounded linear operators from $Y$ to $X$. Assume the following

$$
\begin{aligned}
& G\left(x^{*}\right)=0, G^{\prime}\left(x^{*}\right)^{-1} \in B L(Y, X) \\
& \left\|G^{\prime}\left(x^{*}\right)^{-1}\left(G^{\prime}(x)-G^{\prime}\left(x^{*}\right)\right)\right\| \leq M_{1}\left\|x-x^{*}\right\|^{p} \\
& \left\|G^{\prime}\left(x^{*}\right)^{-1}\left(G^{\prime}(x)-G^{\prime}(y)\right)\right\| \leq M_{2}\|x-y\|^{p}
\end{aligned}
$$

Usually, a third assumption is used that can be written as

$$
\left\|G^{\prime}\left(x^{*}\right)^{-1} G^{\prime}(x)\right\| \leq M, \forall x \in B\left(x^{*}, \frac{1}{M_{1}^{1 / p}}\right)
$$

for $M>0$ in several papers $[7,13]$. We have not used this condition in our work and found that larger radius of the convergence balls are obtained.

Lemma 1 If $G$ satisfies (2.10) and (2.11), then for all $x \in D, p \in(0,1]$ and $t \in[0,1]$, we get

$$
\begin{aligned}
\left\|G^{\prime}\left(x^{*}\right)^{-1} G^{\prime}(x)\right\| & \leq 1+M_{1}\left\|x-x^{*}\right\|^{p}, \\
\| G^{\prime}\left(x^{*}\right)^{-1}\left(G^{\prime}\left(x^{*}+t\left(x-x^{*}\right)\right) \|\right. & \leq 1+M_{1}\left\|x-x^{*}\right\|^{p}, \\
\left\|G^{\prime}\left(x^{*}\right)^{-1} G(x)\right\| & \leq\left(1+M_{1}\left\|x-x^{*}\right\|^{p}\right)\left\|x-x^{*}\right\| .
\end{aligned}
$$

Proof Using (2.10), we get

$$
\left\|G^{\prime}\left(x^{*}\right)^{-1} G^{\prime}(x)\right\| \leq 1+\left\|G^{\prime}\left(x^{*}\right)^{-1}\left(G^{\prime}(x)-G^{\prime}\left(x^{*}\right)\right)\right\| \leq 1+M_{1}\left\|x-x^{*}\right\|^{p} .
$$

and then

$$
\left\|G^{\prime}\left(x^{*}\right)^{-1}\left(G^{\prime}\left(x^{*}+t\left(x-x^{*}\right)\right)\right)\right\| \leq 1+M_{1} t^{p}\left\|x-x^{*}\right\|^{p} \leq 1+M_{1}\left\|x-x^{*}\right\|^{p} .
$$

To prove (2.15), we use mean value theorem, we get

$$
\begin{aligned}
\left\|G^{\prime}\left(x^{*}\right)^{-1} G(x)\right\| & =\left\|G^{\prime}\left(x^{*}\right)^{-1}\left(G(x)-G\left(x^{*}\right)\right)\right\| \leq\left\|G^{\prime}\left(x^{*}\right)^{-1} G^{\prime}\left(x^{*}+t\left(x-x^{*}\right)\right)\left(x-x^{*}\right)\right\| \\
& \leq\left(\left\|1+M_{1}\right\| x-x^{*} \|^{p}\right)\left\|x-x^{*}\right\| .
\end{aligned}
$$

The following theorem gives the local convergence of $(2.7)$.

Theorem 1 Let $x^{*} \in D$ and $\left.a \in\right] \frac{4}{5}, \frac{5}{4}\left[\right.$ such that (2.10)-(2.11) are satisfied and $\bar{B}\left(x^{*}, \rho\right) \subseteq D$, where, the radius $\rho$ is to be determined. Starting with $x_{0} \in B\left(x^{*}, \rho\right)$, the sequence $\left\{x_{k}\right\}$ generated from (2.7) belongs to $B\left(x^{*}, \rho\right)$ and converges to $x^{*}$. The following hold for $k \geq 0$

$$
\begin{aligned}
\left\|y_{k}-x^{*}\right\| & \leq l_{1}\left(\left\|x_{k}-x^{*}\right\|\right)\left\|x_{k}-x^{*}\right\|<\left\|x_{k}-x^{*}\right\|<\rho, \\
\left\|z_{k}-x^{*}\right\| & \leq l_{2}\left(\left\|x_{k}-x^{*}\right\|\right)\left\|x_{k}-x^{*}\right\|<\left\|x_{k}-x^{*}\right\|<\rho, \\
\left\|x_{k+1}-x^{*}\right\| & \leq l_{3}\left(\left\|x_{k}-x^{*}\right\|\right)\left\|x_{k}-x^{*}\right\|<\left\|x_{k}-x^{*}\right\|<\rho,
\end{aligned}
$$

where the functions $l_{1}, l_{2}$ and $l_{3}$ are to be defined. If there exists $R \in\left[\rho,\left(\frac{1+p}{M_{1}}\right)^{1 / p}\right)$ such that $\bar{B}\left(x^{*}, R\right) \subseteq D$, then $x^{*}$ is unique in $\bar{B}\left(x^{*}, R\right)$. 
Proof Using (2.10) and suppose that $\left\|x_{0}-x^{*}\right\|^{p}<\frac{1}{M_{1}}$, then

$$
\left\|G^{\prime}\left(x^{*}\right)^{-1}\left(G^{\prime}\left(x_{0}\right)-G^{\prime}\left(x^{*}\right)\right)\right\| \leq M_{1}\left\|x_{0}-x^{*}\right\|^{p}<1 .
$$

Thus, by Banach Lemma, $G^{\prime}\left(x_{0}\right)^{-1}$ exists and

$$
\left\|G^{\prime}\left(x_{0}\right)^{-1} G^{\prime}\left(x^{*}\right)\right\| \leq \frac{1}{1-M_{1}\left\|x_{0}-x^{*}\right\|^{p}}
$$

Therefore, $y_{0}$ and $z_{0}$ are well defined. For $k=0,(2.7)$ gives

$$
\begin{aligned}
y_{0}-x^{*} & =-G^{\prime}\left(x_{0}\right)^{-1}\left(G\left(x_{0}\right)-G^{\prime}\left(x_{0}\right)\left(x_{0}-x^{*}\right)\right)+(1-a) G^{\prime}\left(x_{0}\right)^{-1} G\left(x_{0}\right) \\
& =-G^{\prime}\left(x_{0}\right)^{-1} G^{\prime}\left(x^{*}\right) \int_{0}^{1} G^{\prime}\left(x^{*}\right)^{-1}\left[G^{\prime}\left(x^{*}+t\left(x_{0}-x^{*}\right)\right)-G^{\prime}\left(x_{0}\right)\right]\left(x_{0}-x^{*}\right) d t \\
& +(1-a) G^{\prime}\left(x_{0}\right)^{-1} G^{\prime}\left(x^{*}\right) \int_{0}^{1} G^{\prime}\left(x^{*}\right)^{-1} G^{\prime}\left(x^{*}+t\left(x_{0}-x^{*}\right)\right)\left(x_{0}-x^{*}\right) d t
\end{aligned}
$$

Taking norm and using (2.11) and (2.19), we get

$$
\begin{aligned}
\left\|y_{0}-x^{*}\right\| & \leq\left\|G^{\prime}\left(x_{0}\right)^{-1} G^{\prime}\left(x^{*}\right)\right\|\left\|\int_{0}^{1} G^{\prime}\left(x^{*}\right)^{-1}\left[G^{\prime}\left(x^{*}+t\left(x_{0}-x^{*}\right)\right)-G^{\prime}\left(x_{0}\right)\right]\left(x_{0}-x^{*}\right) d t\right\| \\
& +|1-a|\left\|G^{\prime}\left(x_{0}\right)^{-1} G^{\prime}\left(x^{*}\right)\right\|\left\|\int_{0}^{1} G^{\prime}\left(x^{*}\right)^{-1} G^{\prime}\left(x^{*}+t\left(x_{0}-x^{*}\right)\right)\left(x_{0}-x^{*}\right) d t\right\| \\
& \leq \frac{1}{1-M_{1}\left\|x_{0}-x^{*}\right\|^{p}}\left[\frac{M_{2}}{1+p}\left\|x_{0}-x^{*}\right\|^{p}+|1-a|\left(1+M_{1}\left\|x_{0}-x^{*}\right\|^{p}\right)\right]\left\|x_{0}-x^{*}\right\| \\
& =l_{1}\left(\left\|x_{0}-x^{*}\right\|\right)\left\|x_{0}-x^{*}\right\|,
\end{aligned}
$$

where,

$$
l_{1}(u)=\frac{1}{1-M_{1} u^{p}}\left(\frac{M_{2}}{1+p} u^{p}+|1-a|\left(1+M_{1} u^{p}\right)\right) .
$$

Let $m_{1}(u)=l_{1}(u)-1$. Then, $m_{1}(0)=|1-a|-1<0$ if $a \in(0,2)$ and $m_{1}\left(\left(\frac{1}{M_{1}}\right)^{1 / p}\right) \rightarrow+\infty$. Therefore, $m_{1}(u)$ has at least one root in $\left(0,\left(\frac{1}{M_{1}}\right)^{1 / p}\right)$ and let $\rho_{1}$ be the smallest root. Thus

$$
0<\rho_{1}<\left(\frac{1}{M_{1}}\right)^{1 / p}
$$

and

$$
0 \leq l_{1}(u)<1, \forall \quad u \in\left[0, \rho_{1}\right) .
$$

Therefore, by using (2.20) and (2.22), we get

$$
\left\|y_{0}-x^{*}\right\| \leq l_{1}\left(\left\|x_{0}-x^{*}\right\|\right)\left\|x_{0}-x^{*}\right\|<\left\|x_{0}-x^{*}\right\| .
$$

Again, from (2.7) for $k=0$, and using (2.15) and (2.19), we get

$$
\begin{aligned}
\left\|z_{0}-x^{*}\right\| & \leq\left\|y_{0}-x^{*}\right\|+\left\|G^{\prime}\left(x_{0}\right)^{-1} G^{\prime}\left(x^{*}\right)\right\|\left\|G^{\prime}\left(x^{*}\right)^{-1} G\left(y_{0}\right)\right\|, \\
& \leq\left(1+\frac{1+M_{1}\left\|y_{0}-x^{*}\right\|^{p}}{1-M_{1}\left\|x_{0}-x^{*}\right\|^{p}}\right)\left\|y_{0}-x^{*}\right\| \\
& \leq\left(1+\frac{\left.1+M_{1}\left(l_{1}\left(\left\|x_{0}-x^{*}\right\|\right)\right)^{p}\left\|x_{0}-x^{*}\right\|^{p}\right)}{1-M_{1}\left\|x_{0}-x^{*}\right\|^{p}}\right) l_{1}\left(\left\|x_{0}-x^{*}\right\|\right)\left\|x_{0}-x^{*}\right\| \\
& =l_{2}\left(\left\|x_{0}-x^{*}\right\|\right)\left\|x_{0}-x^{*}\right\|,
\end{aligned}
$$


where,

$$
l_{2}(u)=\left(1+\frac{1+M_{1}\left(l_{1}(u)\right)^{p} u^{p}}{1-M_{1} u^{p}}\right) l_{1}(u) .
$$

Let $m_{2}(u)=l_{2}(u)-1$. Then, $m_{2}(0)=2|1-a|-1<0$ if $a \in\left(\frac{1}{2}, \frac{3}{2}\right)$ and $m_{2}\left(\rho_{1}\right)=\frac{1+M_{1} \rho_{1}^{p}}{1-M_{1} \rho_{1}^{p}}>0$. Therefore, $m_{2}(u)$ has at least one root in $\left(0, \rho_{1}\right)$ and let $\rho_{2}$ be the smallest root. Thus,

$$
0<\rho_{2}<\rho_{1}
$$

and

$$
0 \leq l_{2}(u)<1, \forall u \in\left[0, \rho_{2}\right)
$$

Using (2.23) and (2.25), we get

$$
\left\|z_{0}-x^{*}\right\| \leq l_{2}\left(\left\|x_{0}-x^{*}\right\|\right)\left\|x_{0}-x^{*}\right\|<\left\|x_{0}-x^{*}\right\| .
$$

Using $y_{0} \in D$ and (2.10), we get

$$
\left\|G^{\prime}\left(x^{*}\right)^{-1}\left(G^{\prime}\left(y_{0}\right)-G^{\prime}\left(x^{*}\right)\right)\right\| \leq M_{1}\left\|y_{0}-x^{*}\right\|^{p} \leq M_{1}\left\|x_{0}-x^{*}\right\|^{p}<1,
$$

Therefore, by Banach Lemma, $G^{\prime}\left(y_{0}\right)^{-1}$ exists and

$$
\left\|G^{\prime}\left(y_{0}\right)^{-1} G^{\prime}\left(x^{*}\right)\right\| \leq \frac{1}{1-M_{1}\left\|y_{0}-x^{*}\right\|^{p}} .
$$

Therefore,

$$
\begin{aligned}
\left\|x_{1}-x^{*}\right\| \leq & \left\|z_{0}-x^{*}\right\|+\left\|\left(\frac{1}{a} G^{\prime}\left(y_{0}\right)^{-1}+\left(1-\frac{1}{a}\right) G^{\prime}\left(x_{0}\right)^{-1}\right) G\left(z_{0}\right)\right\| \\
\leq & \left\|z_{0}-x^{*}\right\|+\left(\frac{1}{|a|}\left\|G^{\prime}\left(y_{0}\right)^{-1} G^{\prime}\left(x^{*}\right)\right\|+\left|1-\frac{1}{a}\right|\left\|G^{\prime}\left(x_{0}\right)^{-1} G^{\prime}\left(x^{*}\right)\right\|\right)\left\|G^{\prime}\left(x^{*}\right)^{-1} G\left(z_{0}\right)\right\| \\
\leq & {\left[1+\left(\frac{1}{|a|} \frac{1}{1-M_{1}\left\|y_{0}-x^{*}\right\|^{p}}+\left|1-\frac{1}{a}\right| \frac{1}{1-M_{1}\left\|x_{0}-x^{*}\right\|^{p}}\right)\right.} \\
& \left.\left(1+M_{1}\left\|z_{0}-x^{*}\right\|^{p}\right)\right]\left\|z_{0}-x^{*}\right\| \\
\leq & {\left[1+\left(\frac{1}{|a|} \frac{1}{1-M_{1}\left\|y_{0}-x^{*}\right\|^{p}}+\left|1-\frac{1}{a}\right| \frac{1}{1-M_{1}\left\|x_{0}-x^{*}\right\|^{p}}\right)\right.} \\
& \left.\left(1+M_{1}\left(l_{2}\left(\left\|x_{0}-x^{*}\right\|\right)^{p}\left\|x_{0}-x^{*}\right\|^{p}\right)\right)\right] l_{2}\left(\left\|x_{0}-x^{*}\right\|\right)\left\|x_{0}-x^{*}\right\| \\
= & l_{3}\left(\left\|x_{0}-x^{*}\right\|\right)\left\|x_{0}-x^{*}\right\|
\end{aligned}
$$

where,

$$
l_{3}(u)=\left[1+\left(\frac{1}{|a|} \frac{1}{1-M_{1}\left(l_{1}(u)\right)^{p} u^{p}}+\left|1-\frac{1}{a}\right| \frac{1}{1-M_{1} u^{p}}\right)\left(1+M_{1}\left(l_{2}(u)\right)^{p} u^{p}\right)\right] l_{2}(u) .
$$

Let $m_{3}(u)=l_{3}(u)-1$. Then,

$$
m_{3}(0)=\left[1+\left(\frac{1}{|a|}+\left|1-\frac{1}{a}\right|\right)\right] l_{2}(0)-1<0
$$


if $a \in] \frac{4}{5}, \frac{5}{4}\left[\right.$ and $m_{3}\left(\rho_{2}\right)=\left[\left(\frac{1}{|a|} \frac{1}{1-M_{1}\left(l_{1}\left(\rho_{2}\right)\right)^{p} \rho_{2}^{p}}+\left|1-\frac{1}{a}\right| \frac{1}{1-M_{1} \rho_{2}^{p}}\right)\left(1+M_{1} \rho_{2}^{p}\right)\right]>0$. Therefore, $m_{3}(u)$ has at least one root in $\left(0, \rho_{2}\right)$ and let $\rho$ be the smallest root. Therefore,

$$
\rho<\rho_{2}<\rho_{1}<\left(\frac{1}{M_{1}}\right)^{1 / p}
$$

and

$$
0 \leq l_{3}(u)<1, \forall \quad u \in[0, \rho)
$$

Therefore, for $a \in] \frac{4}{5}, \frac{5}{4}[$, we have

$$
0<\rho<\rho_{2}<\rho_{1}<\left(\frac{1}{M_{1}}\right)^{1 / p}
$$

By using (2.27) and (2.30), we have

$$
\left\|x_{1}-x^{*}\right\| \leq l_{3}\left(\left\|x_{0}-x^{*}\right\|\right)\left\|x_{0}-x^{*}\right\|<\left\|x_{0}-x^{*}\right\|<\rho .
$$

Therefore, the theorem holds for $k=0$. Replacing $x_{0}, y_{0}, z_{0}$ and $x_{1}$ by $x_{k}, y_{k}, z_{k}, x_{k+1}$ in the similar way, we get the inequalities (2.16)-(2.18) for $k=0,1,2, \ldots$. Since $\left\|x_{k+1}-x^{*}\right\| \leq \| x_{k}-$ $x^{*} \|<\rho$, we get $x_{k+1} \in B\left(x^{*}, \rho\right)$. Since $l_{3}(u)$ is an increasing function in $[0, \rho)$, so we have

$$
\begin{aligned}
\left\|x_{k+1}-x^{*}\right\| & \leq l_{3}(u)\left\|x_{k}-x^{*}\right\| \leq l_{3}(u) l_{3}\left(\left\|x_{k-1}-x^{*}\right\|\right)\left\|x_{k-1}-x^{*}\right\| \\
& \leq l_{3}(u)^{2} l_{3}\left(\left\|x_{k-2}-x^{*}\right\|\right)\left\|x_{k-2}-x^{*}\right\| \leq \ldots \leq l_{3}(u)^{k+1}\left\|x_{0}-x^{*}\right\| .
\end{aligned}
$$

Since $l_{3}(u)<1 \forall u \in[0, \rho)$. Therefore, $\lim _{k \rightarrow \infty} x_{k}=x^{*}$ as $\lim _{k \rightarrow \infty} l_{3}(u)^{k+1}=0$. To prove uniqueness part, let $y^{*} \in B\left(x^{*}, \rho\right), y^{*} \neq x^{*}$ with $G\left(y^{*}\right)=0$. Let $T=\int_{0}^{1} G^{\prime}\left(y^{*}+t\left(x^{*}-y^{*}\right)\right) d t$. Using $(2.10)$, we get

$\left\|G^{\prime}\left(x^{*}\right)^{-1}\left(T-G^{\prime}\left(x^{*}\right)\right)\right\| \leq \int_{0}^{1} M_{1}\left\|y^{*}+t\left(x^{*}-y^{*}\right)-x^{*}\right\|^{p} d t \leq \frac{M_{1}}{1+p}\left\|x^{*}-y^{*}\right\|^{p}=\frac{M_{1}}{1+p} R^{p}<1$,

therefore, $T^{-1}$ exists by Banach Lemma. Then, from the identity

$$
0=G\left(x^{*}\right)-G\left(y^{*}\right)=T\left(x^{*}-y^{*}\right)
$$

we obtain $x^{*}=y^{*}$.

\section{Numerical examples}

In this section, examples are solved to demonstrate the applicability of our methodology.

Example 2 Consider the function $G$ defined on $D=\left[-\frac{1}{2}, \frac{5}{2}\right]$ by

$$
G(x)= \begin{cases}x^{3} \ln x^{2}+x^{5}-x^{4}, & x \neq 0 \\ 0, & x=0\end{cases}
$$


Table 1: Values of parameters

\begin{tabular}{llll}
\hline Examples & $a$ & $\lambda$ & $\alpha$ \\
\hline 2 & 1 & 0.75 & -0.9951 \\
3 & 1 & 0.5 & -1 \\
4 & 0.987 & 0.05 & -1.5 \\
5 & 1 & 0.5 & -1 \\
\hline
\end{tabular}

The derivatives of $G$ are

$$
\begin{aligned}
G^{\prime}(x) & =3 x^{2} \ln x^{2}+5 x^{4}-4 x^{3}+2 x^{2}, \\
G^{\prime \prime}(x) & =6 x \ln x^{2}+20 x^{3}-12 x^{2}+10 x, \\
G^{\prime \prime \prime}(x) & =6 \ln x^{2}+60 x^{2}-24 x+22 .
\end{aligned}
$$

Clearly, $G^{\prime \prime \prime}$ is unbounded on $D$. By applying Theorem 1 with $x^{*}=1$ and $p=1$, we get $M_{1}=M_{2}=96.6628$. Taking $a=1$, we get

$$
\rho=0.002818<\rho_{2}=0.004205<\rho_{1}=0.006896 .
$$

Example 3 Consider the nonlinear Hammerstein type integral equation given by

$$
G(x)(s)=x(s)-5 \int_{0}^{1} s t x(t)^{3 / 2} d t
$$

with $x(s)$ in $\mathcal{C}[0,1]$.

So, we obtain $p=0.5, x^{*}=0, M_{1}=\frac{15}{4}$ and $M_{2}=\frac{15}{4}$. By applying Theorem 1 with $a=1$, we get

$$
\rho=0.003342<\rho_{2}=0.008489<\rho_{1}=0.025599 .
$$

Example 4 Define $G$ on $D=[1,3]$ by

$$
G(x)=\frac{2}{3} x^{\frac{3}{2}}-x
$$

Then, $x^{*}=\frac{9}{4}, p=0.5, G^{\prime}\left(x^{*}\right)^{-1}=2, M_{1}=1$ and $M_{2}=2$. Taking $a=0.9870$, we get

$$
\rho=0.015674<\rho_{2}=0.050026<\rho_{1}=0.176951 .
$$

Example 5 Consider the function $G$ defined on $D=\bar{B}(0,1)$ for $x=\left(x_{1}, x_{2}, x_{3}\right)^{t}$ by

$$
G(x)=\left(e^{x_{1}}-1, \frac{e-1}{2} x_{2}^{2}+x_{2}, x_{3}\right)^{t} .
$$

For $x^{*}=(0,0,0)$, we obtained $K_{0}=e-1$ and $K_{1}=e$. Taking $a=0.5$, we get

$$
\rho=0.118532<\rho_{2}=0.187710<\rho_{1}=0.324947 .
$$

The parameters used by methods (2.7) and (1.5) are listed in Table 1. We have also compared the radius of convergence ball of present method (2.7) with existing method (1.5) and are listed in Table 2. It can be easily observed that the larger radius of convergence ball is obtained by our approach. 
Table 2: Comparison of radius of a ball

\begin{tabular}{lll}
\multicolumn{3}{c}{ Table 2: Comparison of radius of a ball } \\
\hline 2 & Method $(2.7)$ & Method (1.5) \\
3 & 0.002818 & $1.9 \times 10^{-6}$ \\
4 & $3.3 \times 10^{-3}$ & $5.3 \times 10^{-4}$ \\
5 & 0.015674 & 0.002724 \\
\hline
\end{tabular}

\section{Local convergence analysis with condition 2.12}

In this section, we establish the local convergence of (2.7) with condition (2.12). For this, we define the following functions on the interval $\left[0, \frac{1}{M_{1}^{1 / p}}\right)$ given by

$$
\begin{aligned}
h_{1}(u) & =\frac{1}{1-M_{1} u^{p}}\left(\frac{M_{2}}{1+p} u^{p}+M|1-a|\right) . \\
h_{2}(u) & =\left(1+\frac{1}{1-M_{1} u^{p}}\right) h_{1}(u), \\
& =\left(1+\frac{1}{1-M_{1} u^{p}}\right) \frac{1}{1-M_{1} u^{p}}\left(\frac{M_{2}}{1+p} u^{p}+M|1-a|\right) . \\
h_{3}(u) & =\left(1+M\left(\frac{1}{|a|} \frac{1}{1-M_{1} m_{1}(u)^{p} u^{p}}+\left|1-\frac{1}{a}\right| \frac{1}{1-M_{1} u^{p}}\right)\right) h_{2}(u) . \\
n_{2}(u) & =h_{2}(u)-1 . \\
n_{3}(u) & =h_{3}(u)-1 .
\end{aligned}
$$

Taking $\rho_{1}=\left(\frac{1-M|1-a|}{\frac{M_{2}}{1+p}+M_{1}}\right)^{\frac{1}{p}}$ and $M|1-a|<1$, we get

$$
0 \leq h_{1}(u)<1 \forall u \in\left[0, \rho_{1}\right) .
$$

If $(1+M) M|1-a|<1$, then $n_{2}(0)=(1+M) M|1-a|-1<0$ and

$$
n_{2}\left(\frac{1}{M^{1 / p}}\right)=M\left(\frac{M_{2}}{(1+p) M_{1}^{1 / p}}+M|1-a|\right)>0 .
$$

Therefore, $n_{2}(u)$ has at least one root in $\left(0, \frac{1}{M_{1}^{1 / p}}\right)$. Let $\rho_{2}$ be the smallest root of $n_{2}(u)$. Also $n_{2}\left(\rho_{1}\right)>0$ and $\rho_{1}<\frac{1}{M^{1 / p}}$. Therefore,

$$
0<\rho_{2}<\rho_{1}
$$

and

$$
0 \leq h_{2}(u)<1 \forall u \in\left[0, \rho_{2}\right)
$$

If

$$
\left(1+\frac{M}{|a|}(1+|1-a|)\right)(1+M) M|1-a|<1,
$$


then, $h_{3}(0)<0$ and $h_{3}\left(\rho_{2}\right)>0$. Thus $n_{3}(u)$ has a root in $\left(0, \rho_{2}\right)$. Let $\rho$ be the smallest root of $n_{3}(u)$. Therefore

$$
\rho<\rho_{2}<\rho_{1}
$$

and

$$
0 \leq h_{3}(u)<1 \forall u \in[0, \rho)
$$

The following theorem describes the local convergence of (2.7). The proof is omitted because it is nearly same as Theorem 1

Theorem 2 Let $M_{1}>0, M_{2}>0, M>0$ and $a \in(-\infty, \infty)-0$ be the given parameters. Let $x^{*} \in D$ such that for all $x, y \in D$, the following assumptions hold

$$
\begin{gathered}
M|1-a|<1, \\
(1+M) M|1-a|<1, \\
\left(1+\frac{M}{|a|}(1+|1-a|)\right)(1+M) M|1-a|<1, \\
\left\|G^{\prime}\left(x^{*}\right)^{-1}\left(G^{\prime}(x)-G^{\prime}\left(x^{*}\right)\right)\right\| \leq M_{1}\left\|x-x^{*}\right\|^{p}, \\
\left\|G^{\prime}\left(x^{*}\right)^{-1}\left(G^{\prime}(x)-G^{\prime}(y)\right)\right\| \leq M_{2}\|x-y\|^{p}, \\
\left\|G^{\prime}\left(x^{*}\right)^{-1} G^{\prime}(x)\right\| \leq M \forall x \in B\left(x^{*}, \frac{1}{M_{1}^{1 / p}}\right), B\left(x^{*}, \rho\right) \subseteq D
\end{gathered}
$$

Then, the sequence the $\left\{x_{k}\right\}$ generated by (2.7) belongs to $B\left(x^{*}, \rho\right)$ and converges to $x^{*}$. The following hold for $k \geq 0$

$$
\begin{aligned}
\left\|y_{k}-x^{*}\right\| & \leq h_{1}\left(\left\|x_{k}-x^{*}\right\|\right)\left\|x_{k}-x^{*}\right\|<\left\|x_{k}-x^{*}\right\|<\rho, \\
\left\|z_{k}-x^{*}\right\| & \leq h_{2}\left(\left\|x_{k}-x^{*}\right\|\right)\left\|x_{k}-x^{*}\right\|<\left\|x_{k}-x^{*}\right\|<\rho, \\
\left\|x_{k+1}-x^{*}\right\| & \leq h_{3}\left(\left\|x_{k}-x^{*}\right\|\right)\left\|x_{k}-x^{*}\right\|<\left\|x_{k}-x^{*}\right\|<\rho,
\end{aligned}
$$

If there exists $R \in\left[\rho,\left(\frac{1+p}{M_{1}}\right)^{1 / p}\right)$ such that $\bar{B}\left(x^{*}, R\right) \subseteq D$, then $x^{*}$ is unique in $\bar{B}\left(x^{*}, R\right)$.

Table 3: Comparison of radius of a ball by our method with and without M

\begin{tabular}{llll}
\hline Examples & $M$ & Our method without $M$ & Our method with $M$ \\
\hline 2 & 101.5578 & 0.002818 & $1.9 \times 10^{-6}$ \\
3 & 4.75 & $3.3 \times 10^{-3}$ & $1.23 \times 10^{-4}$ \\
4 & $2(\sqrt{3}-1)$ & 0.015674 & 0.007803 \\
5 & $e$ & 0.118532 & 0.045945 \\
\hline
\end{tabular}

It can be easily observed from the Table 3 that our method gives larger radius of convergence without using $M$ in comparison with those with $M$. However, in special case like $G(x)=\sin x$, we obtain larger radius $\rho=0.28755$ with $M=1$ and $\rho=0.272419$ without $M$ because we use $1+M_{1}\left\|x-x^{*}\right\|^{p}$ as a bound for $M$. 


\section{Conclusions}

Local convergence of a family of iterations for solving nonlinear equations is described using Hölder continuous first derivative in Banach spaces. An existence-uniqueness theorem along with error bounds for the solution is given. Different numerical examples including nonlinear Hammerstein equation are solved. The radii of balls of convergence for them are obtained. A substantial improvement of these radii are found in comparison to some other existing methods under similar conditions for all examples considered.

Acknowledgments The authors thank the referees for their valuable comments which have improved the presentation of the paper. The authors thankfully acknowledge the financial assistance provided by Council of Scientific and Industrial Research (CSIR), New Delhi, India.

\section{References}

1. Cordero, A., Ezquerro, J.A., Hernández-Verón, M.A., Torregrosa, J.R.: On the local convergence of a fifth-order iterative method in Banach spaces. Appl. Math. Comput. 251, 396-403 (2015)

2. Martínez, E., Singh, S., Hueso, J.L., Gupta, D.K.: Enlarging the convergence domain in local convergence studies for iterative methods in Banach spaces. Appl. Math. Comput. 281, 252-265 (2016)

3. Argyros, I.K, Khattri, S.K.: Local convergence for a family of third order methods in Banach spaces. Journal of Mathematics. 46, 53-62 (2014)

4. Argyros, I.K., Hilout, S.: Numerical methods in Nonlinear Analysis. World Scientific Publ. Comp. New Jersey (2013)

5. Argyros, I.K., Hilout, S., Tabatabai, M.A.: Mathematical Modelling with Applications in Biosciences and Engineering, Nova Publishers, New York, (2011)

6. Argyros, I.K., Hilout, A.S.: On the local convergence of fast two-step Newton-like methods for solving nonlinear equations. J. Comput. Appl. Math. 245, 1-9 (2013)

7. Argyros, I.K., George, S., Magreñán, A.A.: Local convergence for multi-point-parametric Chebyshev-Halleytype methods of higher convergence order. J. Comput. Appl. Math. 282, 215-224 (2015)

8. Argyros, I.K., George, S.: Local convergence of modified Halley-like methods with less computation of inversion. Novi. Sad.J. Math. 45, 47-58 (2015)

9. Kantorovich, L.V., Akilov, G.P.: Functional Analysis. Pergamon Press, Oxford (1982)

10. Argyros, I.K., Magreñán, A.A.: A study on the local convergence and dynamics of Chebyshev-Halley-type methods free from second derivative. Numer. Algorithms. 71, 1-23 (2016)

11. Traub, J.F.: Iterative Methods for the Solution of Equations. Prentice-Hall, Englewood Cliffs, New Jersey (1964)

12. D.Li, P.Liu, J.Kou; An improvement ofthe Chebyshev-Halley methods free from second derivative, Appl. Math. Comput. 235 (2014) 221-225.

13. Argyros, I.K., George, S.: Local convergence of deformed Halley method in Banach space under Holder continuity conditions. J. Nonlinear Sci. Appl. 8, 246-254 (2015)

14. Rall, L.B.: Computational solution of nonlinear operator equations. In: Robert E. Krieger (ed.) New York (1979)

15. Xiao, X.Y., Yin, H.W.: Increasing the order of convergence for iterative methods to solve nonlinear systems. (2015) Calcolo DOI 10.1007/s10092-015-0149-9

16. Argyros, I.K., Behl, R., Motsa, S.S.: Local convergence of an efficient high convergence order method using hypothesis only on the first derivative. Algorithms 8, 1076-1087 (2015) 